\title{
UTILIZACIÓN DE HERRAMIENTAS ESTADÍSTICAS PARA EL PROCESAMIENTO DE DATOS DE CAMPO Y LABORATORIO EN MECÁNICA DE ROCAS, CON APLICACIÓN AL SITIO DOS MONTAÑAS, SIQUIRRES, COSTA RICA
}

\author{
Rolando Mora Ch. \\ Escuela Centroamericana de Geología, Universidad de Costa Rica \\ Apartado: 2-14-2060-UCR, San Pedro, San José, Costa Rica. \\ E-mail: rmorach@sol.racsa.co.cr
}

(Recibido 16/3/1999; Aceptado 14/5/1999)

\begin{abstract}
This paper deals with the classification of the rocks of Dos Montañas, using a modification of Deere \& Miller methodology, and with the application of statistic tools to define the confidence intervals of the data. The same type of analysis has been performed to study the results of rigid plate and Goodman jack tests

RESUMEN: En este trabajo se realiza la clasificación de las rocas del sitio Dos Montañas, con la utilización de una modificación de la clasificación de Deere \& Miller y con la aplicación de herramientas estadísticas para establecer el intervalo de confiabilidad de los datos. De la misma manera se han estudiado los módulos de esfuerzo-deformación, obtenidos en los ensayos de placa rígida y ensayos en perforaciones (Goodman jack).
\end{abstract}

\section{INTRODUCCIÓN}

Durante la ejecución de ensayos de campo y laboratorio en mecánica de rocas se genera una enorme cantidad de resultados, los cuales muchas veces son aceptados como correctos sin tener en cuenta ningún criterio para diferenciarlos o descartarlos. En la mayoría de los casos se les aplican cálculos estadísticos descriptivos básicos y no se tiene en cuenta la confiabilidad de los resultados.

Es por lo anterior que en este trabajo se pretende introducir una metodología estadística sencilla, con la que se establecen los intervalos de confiabilidad de los datos y se logran discriminar los efectos causados por el ensayo de litologías diferentes, y la aplicación de presiones en sentidos diferentes también. Así mismo se desean establecer los rangos de variación de los parámetros analizados para cada litología, de manera que sirvan como punto de comparación para ensayos futuros en los mismos u otros tipos de roca.
En este trabajo se han utilizado los resultados de los ensayos de esfuerzo-deformación del sitio Dos Montañas, Siquirres, Costa Rica (Fig. 1). En este lugar el Instituto Costarricense de Electricidad (ICE) ha realizado los estudios correspondientes a la etapa de prefactibilidad para un proyecto hidroeléctrico.

\section{CLASIFICACIÓN SEGÚN RESISTENCIA- DEFORMACIÓN DE LAS ROCAS DEL SITIO DOS MONTAÑAS}

Los resultados de ensayos de compresión inconfinada, con testigos de rocas de las perforaciones realizadas por el Instituto Costarricense de Electricidad (ICE) (Pérez, 1989), se han utilizado para evaluar y clasificar las características de esfuerzo-deformación de las litologías predominantes en el sector de Dos Montañas, Siquirres, donde las brechas lávicas, lavas densas y lavas vesiculares pertenecen a la Unidad de Lavas Alcalinas descrita por Cervantes (1989) y Mora 


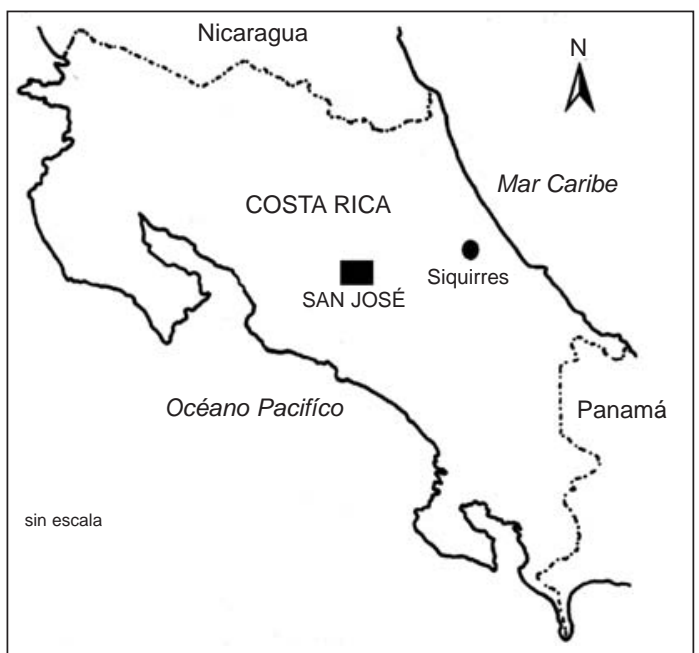

Fig. 1: Localización del área de estudio

\& Cervantes (1993); las areniscas se asocian a la Unidad de Lutitas, Areniscas y Conglomerados, la cual se correlaciona con la Formación Río Banano (Cervantes, 1989; Mora \&Cervantes, 1993).

Se ha utilizado la clasificación de Deere \& Miller (1966), modificándola en el sentido de considerar una categoría más en lo que respecta a la resistencia a la compresión inconfinada, pues como se nota en la figura 2, las areniscas y las brechas lávicas presentan valores inferiores a 10 $\mathrm{MPa}$. La nueva categoría comprende las rocas con resistencia a la compresión inconfinada entre 3 y $10 \mathrm{MPa}$ y se ha denominado como extremadamente baja.

En la figura 2 se observa que al graficar los resultados de resistencia a la compresión inconfinada contra el módulo de esfuerzo-deformación, se obtiene una representación confusa y de dispersión muy marcada, lo cual no permite establecer límites claros y bien definidos entre las litologías. En este sentido se han utilizado algunas herramientas estadísticas para establecer el intervalo de confiabilidad para la media de los resultados de la relación entre el módulo tangente y la resistencia a la compresión inconfinada para cada ensayo.

$\mathrm{Si}$ se consideran las variables como distribuciones normales y se abarcan 1,96 desviaciones estándar a ambos lados de la media, se puede involucrar el $95 \%$ de los resultados, es decir se puede tener un $95 \%$ de certeza de que la media se encontrará en el intervalo así establecido (Lane, 1999). Fuera de este intervalo de confiabilidad

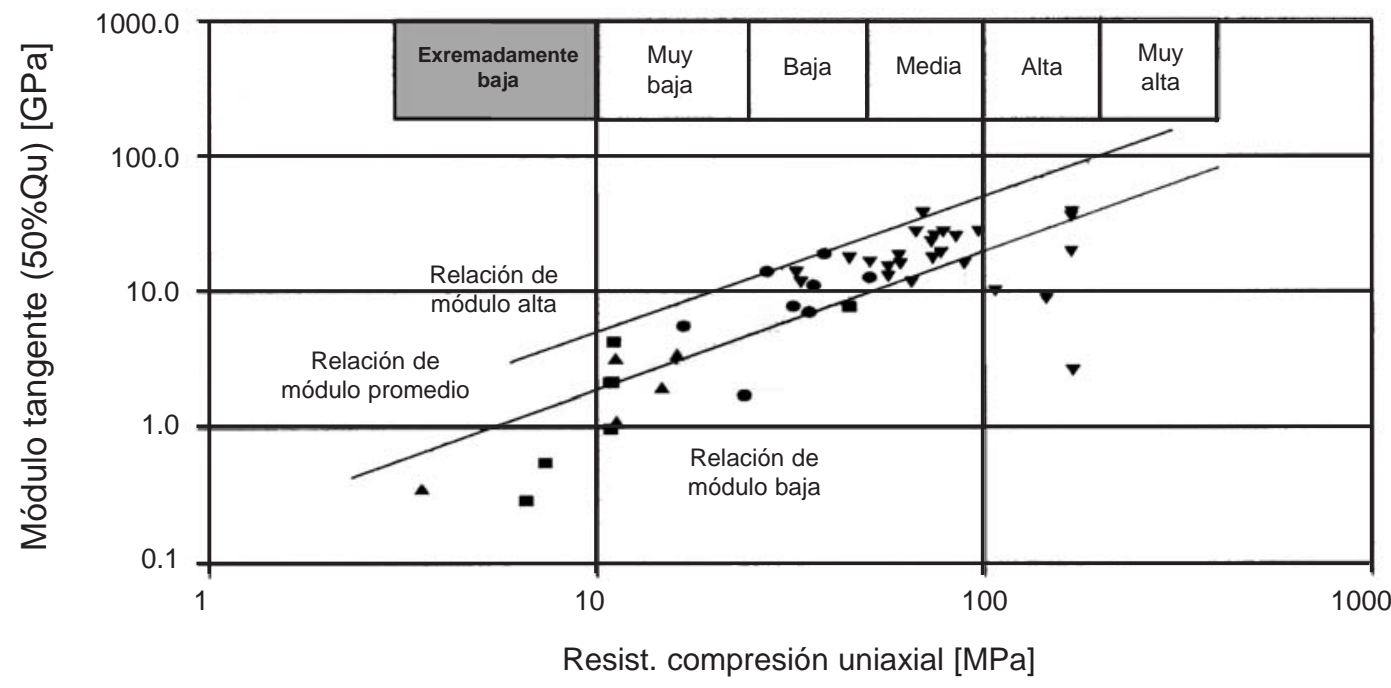

- Brechas lávicas $\quad$ - Lavas densas Lavas vesiculares $\quad$ Areniscas

Fig. 2: Resultados de los ensayos de resistencia a la compresión uniaxial contra los módulos de esfuerzo-deformación, Dos Montañas, Siquirres, Costa Rica. 
las observaciones tienen un $5 \%$ o menos de probabilidades de ocurrencia, es decir los límites del $95 \%$ son un rango de referencia, definen lo que se considera el rango normal (estándar, típico), cualquier cosa fuera de este rango se considera anormal (Swinscow, 1997).

El procedimiento para el cálculo del intervalo de confiabilidad es el siguiente:

- Determinación de la media:

- Determinación del error estándar de la media:

- Utilizar:

$$
\begin{aligned}
& \sigma_{M}=\sigma / \operatorname{SQRT}(N) \\
& z=1,96 \text { para } 95 \% \\
& M-z \sigma_{M} \\
& M+\sigma_{M} \\
& M-z \sigma_{M}<=I C \\
& <=M+z \sigma
\end{aligned}
$$

- Determinación del límite inferior: $\mathrm{M}-\mathrm{z} \sigma_{\mathrm{M}}$

- Determinación del límite superior: $M+\sigma_{M}$

- Intervalo de confiabilidad: $\quad \mathrm{M}-\mathrm{z} \sigma_{\mathrm{M}}<=\mathrm{IC}$

Es así como se ha confeccionado la figura 3 , en la que ahora se definen claramente los ámbitos de cada litología, se han encerrado en un polígono solo los resultados que se encuentran dentro del intervalo de confiabilidad. El resumen de las características de esfuerzo-deformación de las rocas de Dos Montañas se aprecia en el cuadro 1 .
Cuadro 1

\begin{tabular}{|c|c|c|c|}
\hline Litología & $\begin{array}{l}\text { Resistencia a } \\
\text { la compre- } \\
\text { sión uniaxial }\end{array}$ & $\begin{array}{c}\text { Módulo } \\
\text { tangente } \\
(50 \% \mathrm{Qu}) \\
{[\mathrm{GPa}]}\end{array}$ & $\begin{array}{l}\text { Relación de } \\
\text { módulo }\end{array}$ \\
\hline Areniscas & $\begin{array}{l}\text { Extremada- } \\
\text { mente baja a } \\
\text { muy baja } \\
(3-20 \mathrm{MPa})\end{array}$ & $0,3-4,0$ & $\begin{array}{l}\text { Predominan- } \\
\text { temente baja }\end{array}$ \\
\hline $\begin{array}{l}\text { Brechas } \\
\text { lávicas }\end{array}$ & $\begin{array}{c}\text { Muy baja a } \\
\text { baja } \\
(9-50 \mathrm{MPa})\end{array}$ & $0,8-10$ & $\begin{array}{l}\text { De baja a } \\
\text { promedio }\end{array}$ \\
\hline $\begin{array}{l}\text { Lavas } \\
\text { vesiculares }\end{array}$ & $\begin{array}{c}\text { Muy baja a } \\
\text { baja } \\
(15-50 \mathrm{MPa})\end{array}$ & $5-15$ & Promedio \\
\hline Lavas densas & $\begin{array}{c}\text { Media } \\
(50-100 \mathrm{MPa})\end{array}$ & $15-30$ & Promedio \\
\hline
\end{tabular}

Características de esfuerzo-deformación de las rocas de Dos Montañas

\section{MÓDULOS DE ESFUERZO-DEFORMA- CIÓN OBTENIDOS CON EL ENSAYO DE PLACA RÍGIDA}

En este caso se han estudiado únicamente los resultados obtenidos con ensayos realizados en brechas lávicas y lavas sanas; las lavas vesiculares cuentan con un número muy reducido de

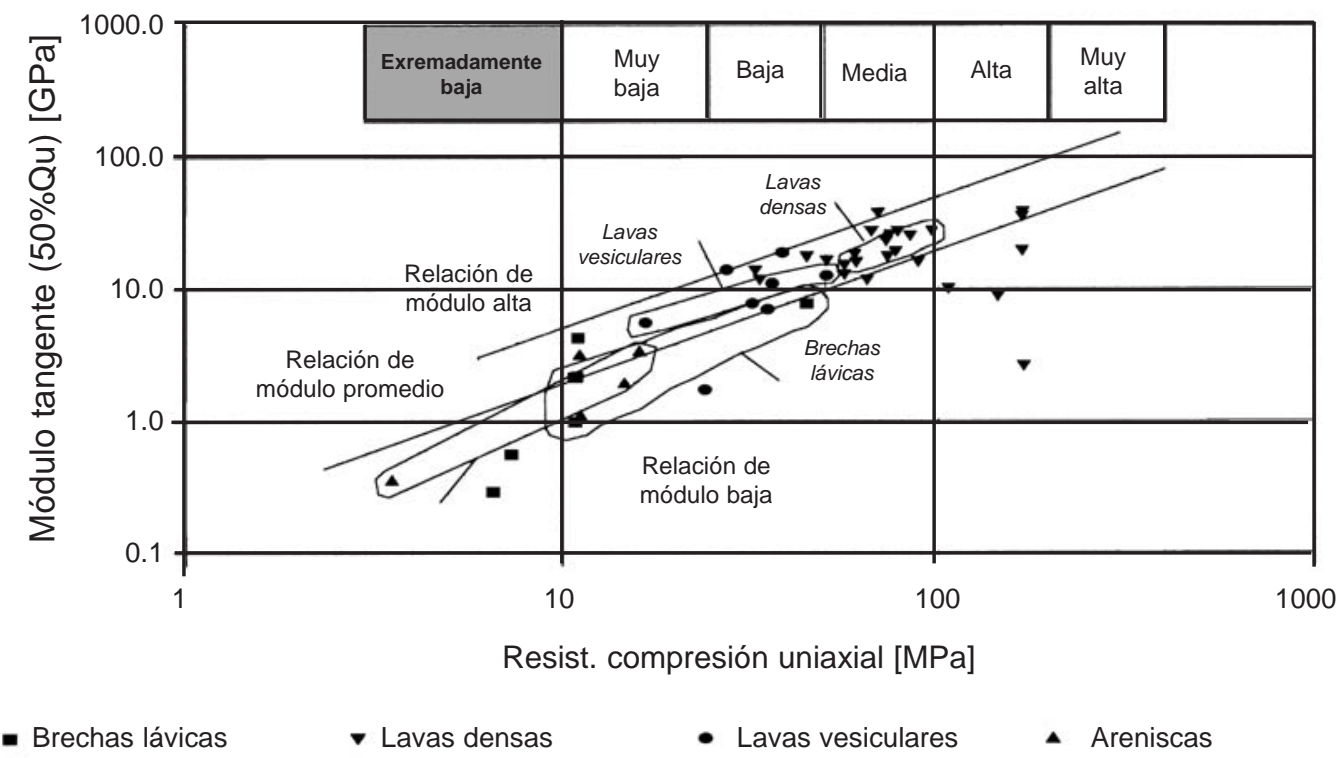

Fig. 3: Definición de los ámbitos litológicos con la ayuda de herramientas estadísticas. Dos Montañas, Siquírres, Costa Rica 
ensayos, lo cual impide un estudio estadístico válido; por otro lado, no se han ejecutado ensayos en las areniscas (Pérez, 1989). Además, se han tomado en cuenta sólo los resultados para los ciclos de carga de 2,9, 4,9 y 6,9 MPa.

Los resultados con las brechas lávicas indican que el módulo de esfuerzo-deformación medido en el techo de la excavación subterránea no difiere sustancialmente del medido en el piso, lo cual sugiere que no se ha desarrollado un fracturamiento significativo del techo, a causa de la excavación de las galerías exploratorias.

Se ha empleado el mismo tratamiento estadístico descrito en el apartado anterior, con el cual se obtiene que los módulos medidos horizontalmente contienen en su rango a los medidos verticalmente (Fig. 4), esto es válido hasta una presión de aproximadamente $5 \mathrm{MPa}$. Lo anterior se puede considerar como un comportamiento isotrópico del material hasta los $5 \mathrm{MPa}$, el cual adopta un comportamiento anisotrópico con el incremento posterior de la presión.

Los módulos promedio en dirección horizontal son mayores que los medidos verticalmente, lo cual indica una mayor capacidad de asumir esfuerzos en dirección horizontal (Cuadro 2), esto probablemente se debe al fracturamiento natural de las brechas lávicas. Por otra parte, los resultados obtenidos con este ensayo se encuentran dentro del rango establecido con los resultados de las pruebas de compresión inconfinada.

Las lavas densas presentan un comportamiento muy similar a las brechas (Fig.5), en el sentido de que los módulos horizontales promedio son mayores que los medidos verticalmente (Cuadro 2). El comportamiento isotrópico se mantiene hasta los $5 \mathrm{MPa}$, luego de esta presión

Cuadro 2

Resumen de los resultados de los ensayos con placa rígida, Dos Montañas

\begin{tabular}{cccc} 
Litología & $\begin{array}{c}\text { Presión de } \\
\text { placa [MPa] }\end{array}$ & $\begin{array}{c}\text { Dirección de } \\
\text { aplicación } \\
\text { del esfuerzo }\end{array}$ & $\begin{array}{c}\text { Módulo de } \\
\text { esfuerzo- } \\
\text { deformación } \\
\text { [GPa] }\end{array}$ \\
Lavas & 2,9 & $\begin{array}{c}\text { Horizontal } \\
\text { Vertical }\end{array}$ & $\begin{array}{c}2,07-5,79 \\
2,29-3,55\end{array}$ \\
densas & 4,9 & Horizontal & $2,54-6,36$ \\
& 6,9 & Vertical & $3,20-4,29$ \\
& & Horizontal & $5,89-8,36$ \\
& & Vertical & - \\
\hline
\end{tabular}

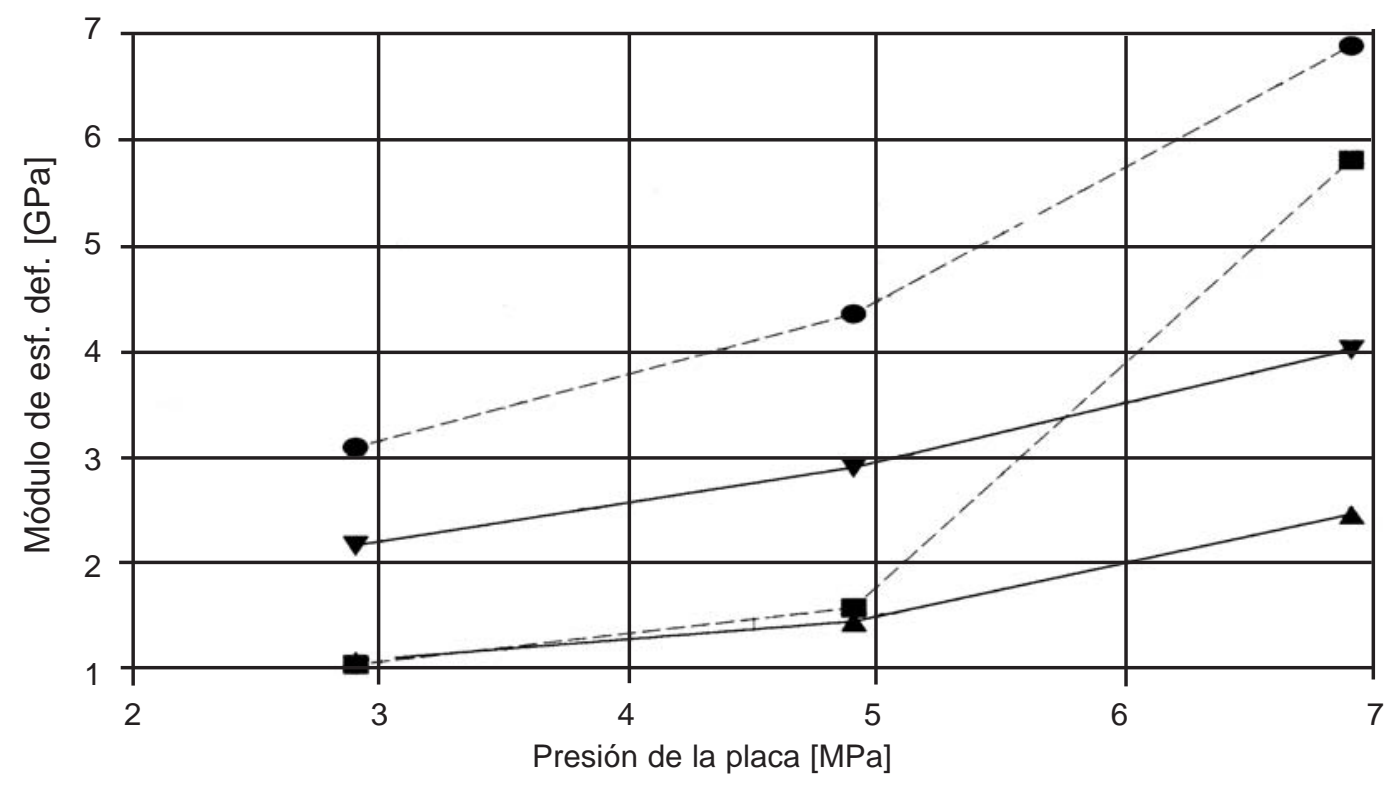

- Horiz. límit. sup. Horiz. lím. inf. $\longrightarrow \quad$ vert. lím. sup. vert. lím. sup.

Fig. 4: Presión de placa contra módulo de esfuerzo-deformación, brechas lávicas, Dos Montañas, Siquirres, Costa Rica. 


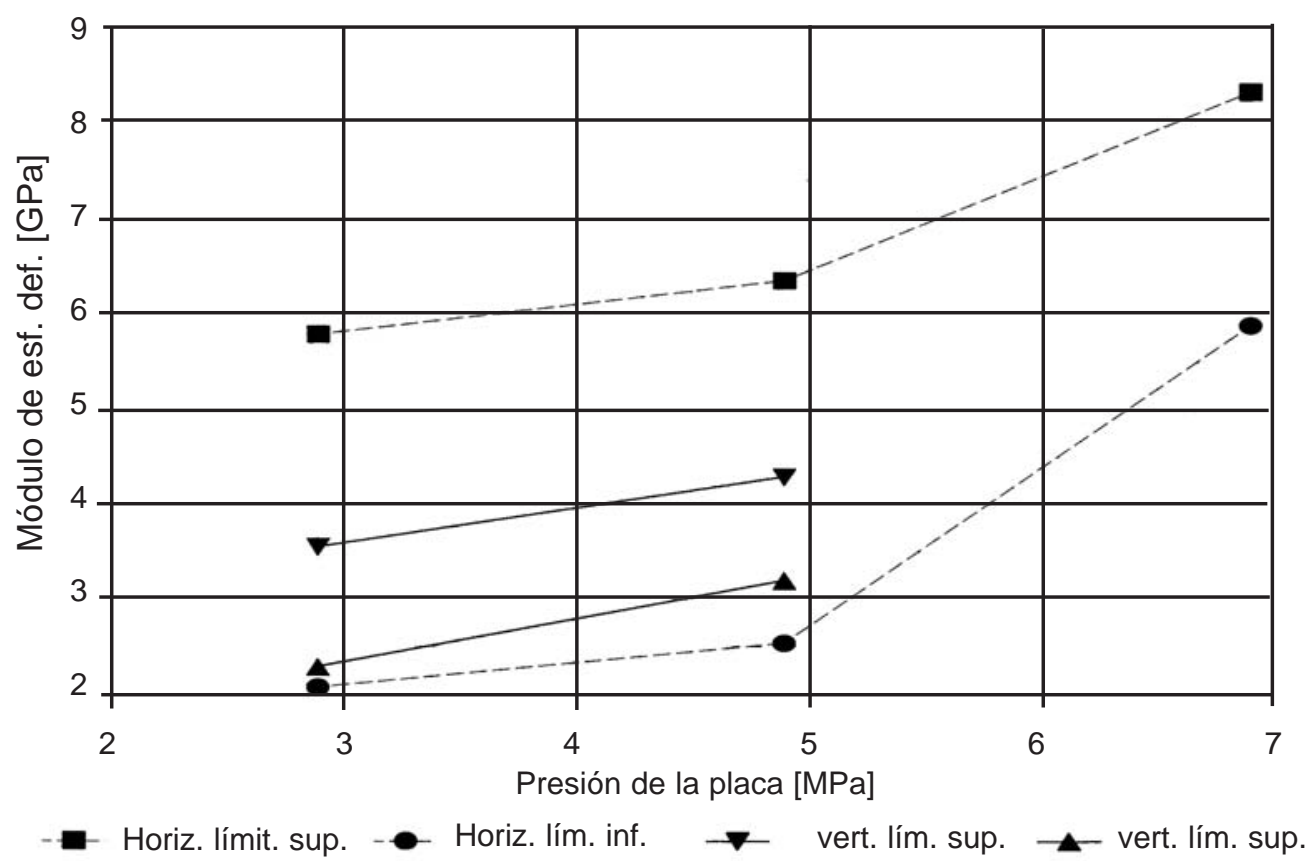

Fig. 5: Presión de placa contra módulo de esfuerzo-deformación, lavas densas, Dos Montañas, Siquirres, Costa Rica.

no se ha podido evaluar la tendencia, pues no se cuenta con el número de ensayos suficiente. La diferencia fundamental estriba en que las lavas densas son capaces de asumir esfuerzos mayores que las brechas lávicas, lo cual es un comportamiento perfectamente normal para estas dos litologías.

Schneider (1967) presenta una clasificación del estado de fracturamiento en rocas, la cual utiliza la relación entre el módulo de esfuerzo-deformación y el módulo envolvente $(\mathrm{E} / \Gamma)$ obtenidos de los ensayos con placa rígida. En la figura 6 se observan los resultados para las brechas lávicas y las lavas densas de Dos Montañas. La delimitación de los dominios se ha ejecutado con la ayuda del tratamiento estadístico previamente descrito. Si el ensayo se realiza en dirección horizontal, las brechas lávicas presentan un comportamiento típico de roca compacta, mientras que las lavas densas se ubican en el espacio para las rocas con fracturas moderadamente abiertas. En caso de que el ensayo se realice en dirección vertical, las brechas lávicas se comportan como rocas con fracturas moderadamente abiertas, y las lavas densas como rocas con fracturas moderadamente abiertas a rocas compactas.

\section{MÓDULOS DE ESFUERZO-DEFORMACIÓN OBTENIDOS CON ENSAYOS EN PERFORACIONES (Goodman jack)}

Se han utilizado los resultados obtenidos para el módulo envolvente final (Pérez, 1989), pues son los que mejor reflejan las diferencias entre litologías, lo cual es útil para tratar de subdividir el macizo rocoso en sectores homogéneos. En el caso de las lavas se determinó que no existe diferencia significativa entre los resultados de las que cuentan con fracturación media y las sanas, cuando el esfuerzo se aplica paralelo al eje propuesto para la presa (Cuadro 3). Si el esfuerzo se aplica perpendicular al eje de presa, las lavas sanas presentan un módulo envolvente final sensiblemente mayor que las de fracturación media y las muy fracturadas (Cuadro 3).

Las brechas lávicas pueden asumir esfuerzos mayores en dirección paralela al eje de presa, 
Cuadro 3

Resumen de resultados para los ensayos realizados en perforaciones, Dos Montañas

\begin{tabular}{ccc}
\hline Litología & $\begin{array}{c}\text { Dirección de apli- } \\
\text { cación del esfuerzo }\end{array}$ & $\begin{array}{c}\text { Módulo envol- } \\
\text { vente final } \\
{[\mathrm{GPa}]}\end{array}$ \\
$\begin{array}{c}\text { Lavas muy } \\
\text { fracturadas }\end{array}$ & $\begin{array}{c}\text { Paralelo } \\
\text { Perpendicular }\end{array}$ & $0,43-0,71$ \\
Lavas de frac- & Paralelo & $2,72-3,30$ \\
turación media & Perpendicular & $4,56-6,36$ \\
a sanas & Paralelo & $1,02-1,93$ \\
Brechas lávicas & Perpendicular & $0,42-1,25$ \\
& Paralelo & $0,77-0,86$ \\
Areniscas & Perpendicular & $1,08-1,12$ \\
\hline
\end{tabular}

que perpendicular al mismo (Cuadro 3). El caso contrario se presenta con las areniscas, donde esta litología puede asumir esfuerzos mayores en dirección perpendicular al eje de presa, que paralelo al mismo (Cuadro 3). En ambos casos, este comportamiento se debe a la orientación preferencial de los sistemas de discontinuidades de las litologías.

\section{CONCLUSIONES}

Luego de la utilización del método estadístico para determinar los intervalos de confiabilidad, se ha notado que una cantidad significativa (en algunos casos más del 50\%) de resultados se han tenido que descartar, pues se localizan fuera del intervalo del 95\% de confiabilidad, lo cual puede significar que la ejecución de los ensayos ha estado caracterizada por errores de instalación o de operación del equipo.

Las areniscas presentan valores de compresión inconfinada inferiores a $10 \mathrm{MPa}$, lo cual conlleva a considerar una categoría de clasificación adicional entre 3 y $10 \mathrm{MPa}$ y que se ha denominado como extremadamente baja.

El empleo de los intervalos de confiabilidad permite eliminar la representación confusa y dispersa de los datos, al mismo tiempo que se establecen límites claros entre las diferentes litologías.

Los ensayos de placa rígida en las brechas lávicas indican que no se presenta fracturamiento por la excavación de las galerías exploratorias,

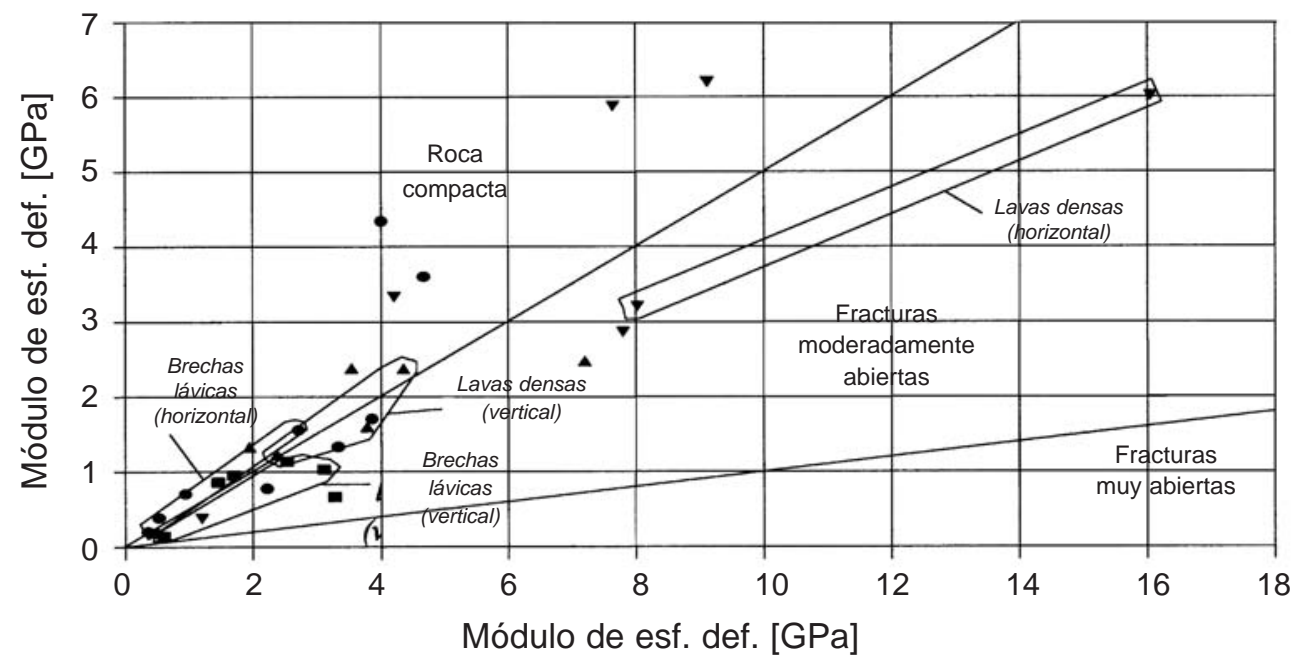

- $\quad$ Brechas lávicas (hrztl)

- Brechas lávicas (vrtcl)

- Lavas densas (hrztl)

- lavas densas (vrtcl)

Fig. 6: Clasificación del estado de fracturamiento de las rocas. 
esto por cuanto los módulos de esfuerzo deformación medidos en el techo y piso no difieren significativamente. Además, el material muestra el fenómeno de anisotropía solo a presiones de placa superiores a los $5 \mathrm{MPa}$, y una capacidad mayor de asumir esfuerzos en dirección horizontal, lo cual tiene su fundamento en el fracturamiento (diaclasamiento) natural de la roca. El comportamiento de las lavas densas es muy similar, solo difiere en éstas que son capaces de asumir esfuerzos mayores que las brechas lávicas.

Las lavas densas y las brechas lávicas pueden clasificarse como de fracturas moderadamente abiertas a rocas compactas, en función de la dirección de aplicación de la presión en el ensayo de placa rígida, sin embargo esto no significa que sean de buena calidad mecánica.

El módulo envolvente final, en los ensayos realizados en perforaciones, varía de acuerdo con la aplicación del esfuerzo con respecto al eje propuesto para la presa y con el tipo de roca ensayada, lo cual es fiel reflejo de las discontinuidades propias de cada material.

\section{REFERENCIAS}

CERVANTES, F., 1989: Base geológica para los análisis geotécnicos del Proyecto
Hidroeléctrico Siquirres. - 102 págs. Univ. de Costa Rica [Tesis Lic.].

DEERE, D.U., \& MILLER, R.P., 1966: Engineering classification and index properties of intact rock. - 77 págs. Air Force Lab.Techn. Rep.Nº. AFNL-TR-65-116, Albuquerque, NM.

LANE, D.M., 1999: HyperStat statistics textbook. - http://www.ruf.rice.edu/ lane/hyperstat/contents.html.

MORA, R. \& CERVANTES, F., 1993: Clasificación geomecánica del macizo rocoso Dos Montañas, Siquirres, Costa Rica. - Rev. Geol. América Central, 16: 39-50.

PÉREZ, C., 1989: Caracterización geológico-geotécnica preliminar del macizo rocoso del Sitio de Presa del Proyecto Hidroeléctrico Siquirres, provincia de Limón, Costa Rica. - 77 págs. Univ. de Costa Rica [Tesis Lic.].

SWINSCOW, T.D.V., 1997 [9 ed.]: Statistics at Square One. - . BMJ Publ. Group. (Internet). 